schaftlichen Nutzung usw.) ergriffen werden.

\section{Danksagung:}

Die Autoren bedanken sich herzlichst bei Dr. A. Havelaar, Bilthoven, für die freundliche Erlaubnis, Abbildung 1 in veränderter Form reproduzieren zu dürfen. Professor D. Kay, Leeds, hat freundlicherweise unveröffentlichtes Material für die Abbildungen 4 und 5 zur Verfügung gestellt. Wesentliche Gedankengänge dieser Arbeit, insbesondere zum Begriff Erkrankungsbeiastung (disease burden) und zur Beziehung der Erkrankungsbelastung zur statistischen Verteilung von Fäkalindikatoren in Gewässern, stammen von ihm.

Frau M. Jung, Berlin, hat die Abbildungen mit großer Sorgfalt angefertigt. Wir danken Frau Dr. C. Höller, Kiel, und Frau Dr. I. Feuerpfeil, Bad Elster, für wertvolle Bemerkungen und Anregungen zum Manuskript.

\section{Literatur:}

[1] Craun, G. F.: Statistics of waterborne outbreaks in the U.S. (1920-1980). Waterborne Diseases in the United States. Boca Raton, Florida: CRC Press, Inc. 1986. 73-159.

[2] Hunter, P. R.: Health effects of bathing in surface waters. Microbiology Europe 4 (1996) 10-12.

[3] Anonymous: Richtlinie des Rates vom 8. Dezember 1975 über die Qualitär der Badegewässer. (76/160/EWG) Amtsblatt der Europäischen Gemeinschaften (1976) L31/1 29-35.

[4] Haas, C. H.: Estimation of risk due to low doses of microorganisms: a comparison of alternative methodologies. Am. J. Epidemiol. 118 (1983) 573-582.

[5] Regli, S., Rose, J. B., Haas, C. N., and Gerba, C. P.: Modelling the risk from giardia and viruses in drinking water. JAWWA (1991) 76-84.

[6] Rose, J. B., and Gerba, C. P.: Assessing potential health risks from viruses and parasites in reclaimed water in Arizona and Florida. Wat. Sci. Technol. 23 (1991) 2091-2098.
[7] Rose, J. B., Haas, C. N., and Regli, S.: Risk assessment and control of waterborne giardiasis. Am. J. Public Hlth. 81 (1991) 709-713.

[8] Rose, J. B., and Sobsey, M. D.: Quantitative risk assessment for viral contamination of shellfish and coastal waters. Journal of Food Protection 56 (1993) 1043-1050.

[9] Rose, J. B., and Gerba, C. P.: Use of risk assessment for development of microbial standards. Wat. Sci. Technol. 24 (1991b) 29-34.

[10] Gerba, C. P., Rose, J. B., Haas, C. N., and Crabtree, K. D.: Waterborne rotavirus: a risk assessment. Wat. Res. 30 (1996) 2929-2940.

[11] Teunis, P. F. M., van der Heijden, O. G., van der Giessen, J. W. B., and Havelaar, A. H.: The dose-response relation in human volunteers for gastro-intestinal pathogens. Report 284550002 from the National Institute of Public Health and the Environment (RIVM) Bilthoven, The Netherlands (1996).

[12] Teunis, P. F. M., Medema, G. J., Kruidenier, L., and Havelaar, A. H.: Assessment of the risk of infection by Cryptosporidium or $\mathrm{Gi}$ ardia in drinking water from a surface wate source. Wat. Res. 31 (1997) 1333-1446.

[13] Kay, D., Fleisher, J. M., Salmon, R. L., Jones, F., Wyer, M. D., Godfree, A. F., ZelenauchJacquotte, Z., and Shore, R.: Predicting likelihood of gastroenteritis from sea bathing: results from randomised exposure. Lancet 344 (1994) 905-909.

[14] Kay, D., Wyer, M., Fleisher, J., and Fewtrell, L.: Technical feasibility of a priori measurement approach for managing bathing wate quality. European Commission Workshops, Sitges, Spain, 1997. Report EUR 17801 EN.

[15] Fleisher, J. M., Jones, F., Kay, D., StanwellSmith, R., Wyer, M., and Morano, R.: Water and non-water-related risk factors for gastroenteritis among bathers exposed to sewage-contaminated marine waters. International Journal of Epidemiology 22 (1993) 698-708.

[16] Fleisher, J. M., Kay, D., Salmon, R. L., Jones, F., Wyer, M. D., and Godfree, A.: Marine Waters Contaminated with Domestic Sewage: Nonenteric Illnesses Associated with Bather
Exposure in the United Kingdom. Am. J. Public Health 86 (1996) 1228-1234.

[17] Ward, L. R., Berstein, D. I., Young, E. C. Sherwood, J. R. Knowlton, D. R., and Schiff, G.M.: Human rotavirus studies in volunteers of infectious dose and serological response to infection. J. Infect. Dis. 154 (1986) 871-880.

[18] Rendtdorff, R. C.: The experimental transmission of human intestinal protozoan parasites 1 . Giardia lamblia cysts given in capsules. Am. J. Hyg. 59 (1954 a) 209 (zitiert aus [11]).

[19] Hornick, R. B., Greisman, S. E., Woodward, T. E., DuPont, H. L., Dawkins, A. T., and Snyder, M. J.: Typhoid fever: pathogenesis and immunologic control. 283 (13) (1970) 686-691 (zitiert aus [11]).

[20] Stevenson, A. H.: Studies of bathing water quality and health, Am. J. Public Hlth 43 (1953) 529-538.

[21] Cabelli, V. J., Dufour, A. P., McCabe, L. J. and Levin, M. A.: Swimming-associated gastroenteritis and water quality. American Journal of Epidemiology 115 (1982) 606-616.

[22] Cabelli, V. J., Dufour, A. P., McCabe, L. J., and Levin, M. A.: A marine recreational water quality criterion consistent with indicator concepts and risk analysis. Journal WPCF 55 (1984) 1306-1314

[23] Pike, E. B.: Statistical aspects of microbial populations in recreational waters. In Kay, D (Hrsg.): Recreational water quality management, Vol. 1: Coastal Waters. Ellis Horwood 1992.

[24] Betzhold, M.: Enteroviren in Berliner Abwasser im Vergleich zu deren Nachweis bei Patienten einiger Berliner Krankenhäuser. Auswertung der Untersuchungsergebnisse des Landesmedizinaluntersuchungsamtes Berlin von Juli 1970 bis Juni 1990. Inaugural-Dissertation, 1993. Freie Universität Berlin.

Anschrift der Verfasser:

Dr. Juan M. López-Pila und Dr. Regine Szewzyk, Institut für Wasser-, Boden- und Lufthygiene des Umweltbundesamtes, Corrensplatz 1, D-14195 Berlin

\title{
Gasbrand - Einzelfallerfassung der Erkrankungs- und Sterbefälle in den neuen Bundesländern und Berlin 1992 bis 1997
}

\section{Einführung}

Gasbranderreger sind ubiquitär im Erdreich, aber auch im Darminhalt vorhanden. Bei Kontakt von Wunden mit Schmutz bzw. Faezes kann es - besonders bei gleichzeitigem Sauerstoffmangel - zu Gasbrand-Infektionen kommen.
Gasbrand tritt vor allem im Zusammenhang mit schlecht durchbluteten Wunden oder offenen Frakturen auf, nach Operationen (insbesondere am MagenDarm-Trakt), bei bestehenden Durchblutungsstörungen (oft in Verbindung mit Diabetes) sowie endogen bei pathologischen Veränderungen des MagenDarm-Traktes (Neoplasmen, Ileus, in- karzerierte Hernien). Seltener wird Gasbrand auch nach Injektionen und nach primär aseptischen Operationen beobachtet.

Gasbranderkrankungen nehmen - von der Zahl der jährlich registrierten Fälle unter den in Deutschland vorkommenden Infektionskrankheiten keinen vor- 


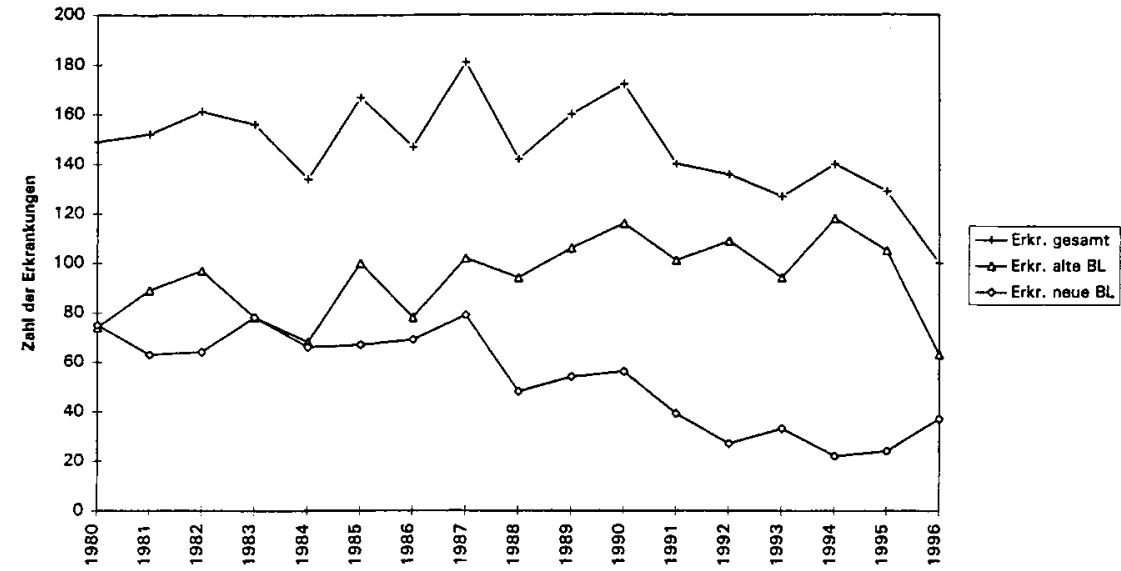

Abbildung 1: Gasbrand - Zahl der Erkrankungen 1980 bis 1996. Zahlen des Statistischen Bundesamtes und Pöhn und Rasch [1].

deren Platz ein. Wegen der noch immer sehr hohen Letalität, die mit den Gasbranderkrankungen verbunden ist, haben diese allerdings eine nicht zu unterschätzende Bedeutung.

Gegenstand dieser Auswertungen soll es deshalb sein, alle im RKI vorliegenden Informationen über in Deutschland in den letzten Jahren aufgetretene Gasbranderkrankungen und -sterbefälle zusammenfassend zu betrachten.

\section{Erkrankungen an Gasbrand in Deutschland}

Während Erkrankungen an Gasbrand im Bundesgebiet erst seit 1980 meldepflichtig sind, liegen Daten für das Gebiet der ehemaligen DDR bereits seit 1955 vor [1].

Im Vergleich zu früheren Jahren blieb die Gesamtzahl der in Deutschland gemeldeten Fälle auf einem annähernd gleichen Niveau, wenngleich es auch insgesamt zu leicht sinkenden Erkrankungszahlen gekommen ist (Abb. 1).
Wurden im Jahre 1989 beispielsweise 160 Erkrankungsfälle erfaßt (106 E in den alten Ländern, $54 \mathrm{E}$ in der DDR) [1], so waren es im Zeitraum von 1992 bis 1996 jährlich 100 bis 140 Erkrankungen. Für das Jahr 1997 liegt die (gegenwärtig noch vorläufige) Zahl der Erkrankungen an Gasbrand bei 122.

Betrachtet man die Zahlen für das Gebiet der neuen Bundesländer, so wird deutlich, daß es in den 90er Jahren im Vergleich zu den 80er Jahren - zu einer Verringerung der Zahl der gemeldeten Gasbrand-Erkrankungen gekommen ist.

Die Zahlen der Sterbefälle, die in der Todesursachenstatistik des Statistischen Bundesamtes ausgewiesen werden, unterlagen in den Jahren von 1992 bis 1996 nur geringen Schwankungen (Abb. 2).

\section{Sondererhebungen}

Für das Gebiet der DDR liegen, beginnend mit dem Jahr 1979, zu allen gemeldeten Gasbrandfällen umfangreiche An- gaben vor. Diese Einzelfallerfassung wurde durch das RKI für das Gebiet der neuen Bundesländer und Berlin im Rahmen von Sondererhebungen fortgesetzt.

Für die nach Bundes-Seuchengesetz aus diesen Ländern gemeldeten Erkrankungs- und Sterbefälle wurden detaillierte Angaben übermittelt, die mit Hilfe eines speziellen Erhebungsbogens erfaßt wurden. In die hier dargestellten Auswertungen wurden alle für den Zeitraum 1992 bis $1997 \mathrm{im} \mathrm{RKI}$ vorliegenden Angaben einbezogen.

In die Auswertungen kamen insgesamt 182 Gasbrandfälle. Die im Rahmen der Einzelfallerfassungen erhobenen Zahlen der Erkrankungs- und Sterbefälle, aufgeschlüsselt auf die Jahre ihres Auftretens, sind in Abbildung 3 dargestellt.

Von den Erkrankungen sind mehr männliche als weibliche Personen betroffen (104 Männer, 77 Frauen, ein Fall ohne Angabe).

Gasbranderkrankungen traten im betrachteten Zeitraum in allen Altersgruppen (mit Ausnahme von Kleinkindern) auf (Abb. 4). Die jüngsten, im Rahmen dieser Sondererhebungen erfaßten Patienten waren ein neunjähriges Mädchen sowie zwei Jungen im Alter von zehn und 14 Jahren. Alle drei erkrankten an Gasbrand nach erlittenen Verletzungen.

Der größte Teil der Fälle wurde jedoch bei Personen höheren Lebensalters registriert. Rund $54 \%$ aller Erkrankungen waren bei Personen, die älter als $65 \mathrm{Jahre}$ alt waren, zu verzeichnen.

In den einzelnen Bundesländern, die in die Sondererhebungen zum Vorkommen von Gasbrand einbezogen waren, kam es in den betrachteten Jahren zu ganz unterschiedlichen Erkrankungszahlen (Tab. 1). Die stark variierenden

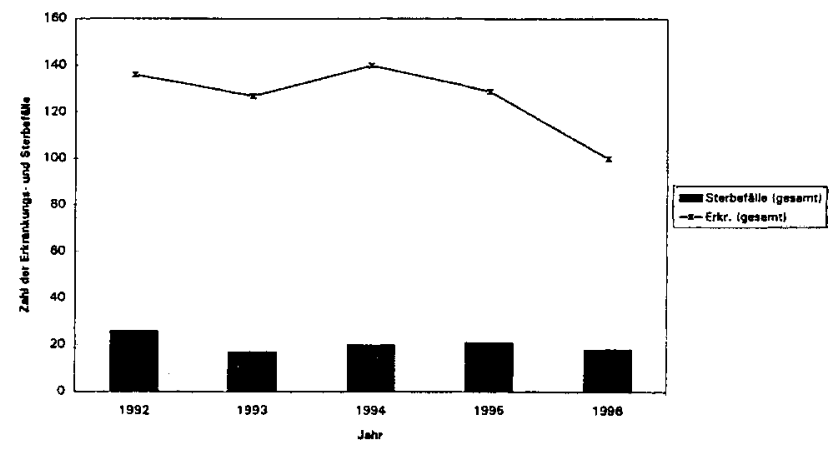

Abbildung 2: Gasbrand - Erkrankungen und Sterbefälle in Deutschland 1992 bis 1996. Zahlen des Statistischen Bundesamtes (Sterbefälle nach Totenscheinanalyse).

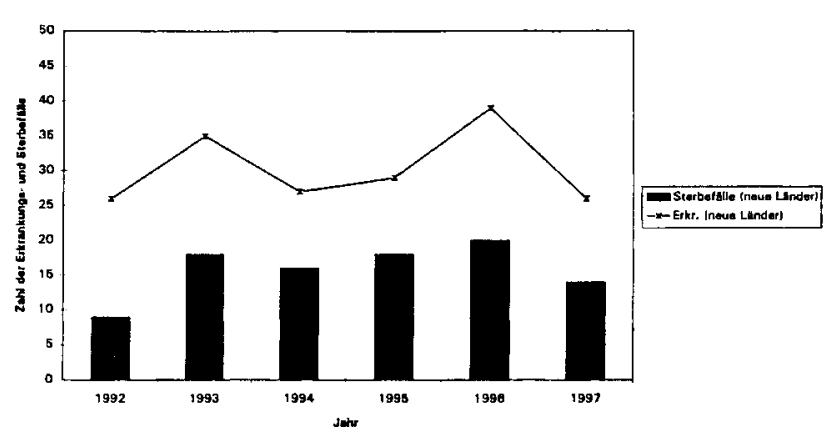

Abbildung 3: Gasbrand - Erkrankungen und Sterbefälle in den neuen Bundesländern und Berlin 1992 bis 1997. Zahlen der Sondererhebung des RKI. 


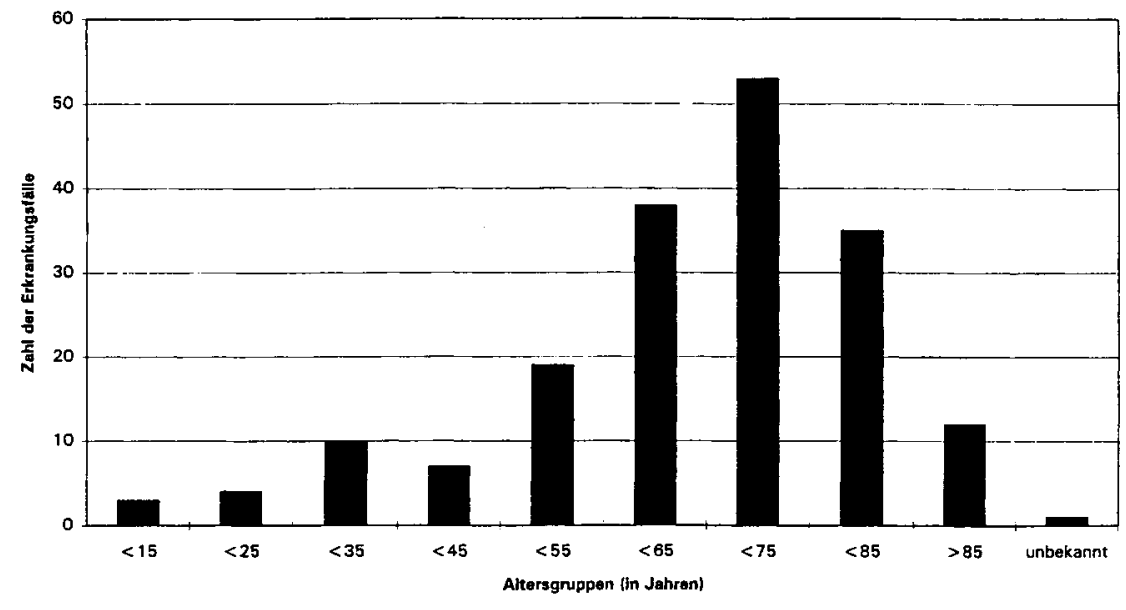

Abbildung 4: Gasbrand in den neuen Bundesländern und Berlin 1992 bis 1997 Altersverteilung.

Zahlen der Erkrankungen, die beispielsweise für Sachsen-Anhalt zwischen elf Erkrankungen im Jahre 1993 und einer Erkrankung 1994 liegen, lassen eine Berechnung von Inzidenzraten für die einzelnen Länder wenig sinnvoll erscheinen.

\section{Ursachen für Gasbrand- erkrankungen}

Aufgrund der schon für viele Jahre vorliegenden Angaben zu den Ursachen der Gasbranderkrankungen wurde eine $\mathrm{Zu}$ ordnung der aufgetretenen Fälle in sechs Gruppen vorgenommen (Abb. 5).

Eine wichtige Gruppe bilden Gasbranderkrankungen, die im Zusammenhang mit dem Magen-Darm-Trakt stehen. Hierin eingeschlossen sind einerseits Operationen an Magen, Darm oder Gallenblase (z. B. Cholezystektomie, Appendektomie), andererseits pathologische Veränderungen des Magen-DarmTraktes (z. B. Neoplasma, Ileus, inkarzerierte Hernien).

Eine weitere Gruppe bilden Gasbranderkrankungen, die in der Folge von Unfällen entstehen. Offene Frakturen, zerstörtes Muskelgewebe oder stark verschmutzte Wunden bieten gute Voraussetzungen für das Entstehen eines Gasbrandes. Aber auch kleinere Verletzungen (z. B. Schürfwunden) können im Ausnahmefall schon zu einer GasbrandErkrankung führen.

Eine wichtige Risikogruppe stellen auch Patienten mit arteriellen Verschlußkrankheiten und Diabetes mellitus dar. Betroffen von den Durchblutungs- störungen sind vorwiegend die unteren Extremitäten, wobei es häufig nach Amputation $\mathrm{zu}$ einem Stumpfgasbrand kommt, der oft eine weitere Amputation nach sich zieht.

Gasbrandfälle nach Injektionen (z. B. nach Insulininjektionen bei selbstspritzenden Diabetikern oder bei Drogenabhängigen) kommen vor, sind jedoch zahlenmäßig heute von geringerer $\mathrm{Be}$ deutung.

Gasbranderkrankungen nach primär aseptischen Operationen (z. B. an Nieren, Hüftgelenk) sind in seltenen Fällen ebenso zu verzeichnen wie Gasbrand nach Dekubitalgeschwüren, Erfrierungen usw.

Die Analyse aller im Zeitraum von 1992 bis 1997 in den neuen Bundesländern aufgetretenen Gasbrand-Erkrankungen - differenziert nach den Hauptrisikogruppen ihrer Entstehung - erbrachte folgende Ergebnisse:

In ca. $40 \%$ der Erkrankungen waren Vorerkrankungen, die mit Durchblutungsstörungen, Diabetes usw. verbun-

Tabelle 1: Erkrankungen an Gasbrand in den neuen Bundesländern und Berlin, differenziert nach Ländern

\begin{tabular}{lccccccc}
\hline Jahr/Land & Berlin & $\begin{array}{l}\text { Branden- } \\
\text { burg }\end{array}$ & $\begin{array}{l}\text { Meckl.- } \\
\text { Vorpom. }\end{array}$ & Sachsen & $\begin{array}{l}\text { Sachsen- } \\
\text { Anhalt }\end{array}$ & Thüringen & Summe \\
\hline 1992 & 4 & 1 & 5 & 4 & 7 & 5 & 26 \\
1993 & 2 & 4 & 3 & 10 & 11 & 5 & 35 \\
1994 & 5 & 8 & 4 & 7 & 1 & 2 & 27 \\
1995 & 5 & 5 & 5 & 4 & 4 & 6 & 29 \\
1996 & 3 & 7 & 7 & 10 & 7 & 5 & 39 \\
1997 & 1 & 5 & 5 & 4 & 4 & 7 & 26 \\
Summe & 20 & 30 & 29 & 39 & 34 & 30 & 182 \\
\hline
\end{tabular}

den waren, bekannt (Abb. 5). In $28 \%$ aller Gasbrandfälle gingen Unfallfolgen (Knochenbrüche, andere Verletzungen) der Erkrankung an Gasbrand voraus. Insgesamt ca. $18 \%$ aller Fälle standen im Zusammenhang mit dem MagenDarm-Trakt, beispielsweise mit Operationen bzw. mit pathologischen Veränderungen in diesem Bereich. Damit ergab sich gegenüber den Auswertungen für einen früheren Zeitraum ein etwas anderes Bild [3], d. h. für die wichtigsten Risikogruppen ergab sich in den letzten Jahren eine Änderung in der Rangfolge.

Waren in den Auswertungen der Jahre 1986 bis 1991 noch Gasbranderkrankungen, deren Entstehung mit dem Magen-Darm-Trakt in Verbindung gebracht werden konnte, an vorderer Stelle (ca. $38 \%$ ), so sind für die betrachteten vergangenen sechs Jahre Verschlußkrankheiten und Diabetes mellitus der wichtigste Risikofaktor. Unfälle nahmen früher (1986 bis 1991) wie heute mit ca. $30 \%$ den zweiten Platz nach Häufigkeit ein.

In diesem Zusammenhang erhebt sich die Frage, ob es sich bei der beobachteten Änderung in der Reihenfolge der Hauptrisikofaktoren tatsächlich um eine Verbesserung der Situation, beispielsweise bei operativen Eingriffen am Magen-Darm-Trakt, handelt oder ob hierfür ein selektiver Meldefehler bzw. eine Untererfassung die Ursache sein könnte.

\section{Sterbefälle}

Bei der Betrachtung der Zahlen der Sterbefälle infolge Gasbrand wird deutlich, $\mathrm{da}$ im Rahmen der Sondererhebungen wesentlich mehr Sterbefälle erfaßt werden, als durch das Statistische Bundesamt für die Jahre 1992 bis 1996 ausgewiesen werden [2]. Das führt beispielsweise dazu, daß in den Jahren 1993 und 1996 mehr Todesfälle über die Sondererhebungen in den neuen Bundeslän- 


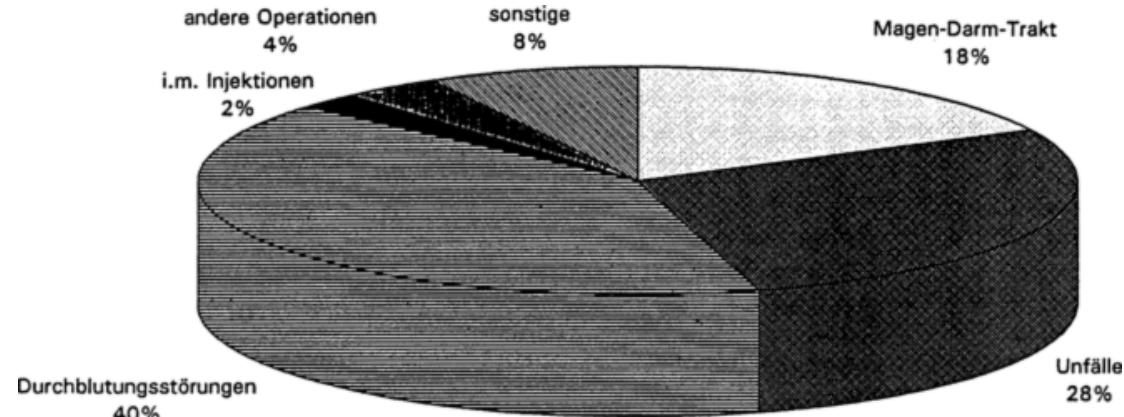
$40 \%$

Abbildung 5: Gasbrand in den neuen Bundesländern und Berlin 1992 bis 1997 - Anteil der Hauptrisikogruppen an der Gesamtzahl der Erkrankungen.

dern erfaßt werden, als für Deutschland insgesamt durch die Bundesstatistik (Abb. 2 und 3).

Ein Vergleich der Sterbefälle, die im Rahmen der Meldungen nach BundesSeuchengesetz und somit durch die Sondererhebungen bzw. durch das Statistische Bundesamt für die neuen Bundesländer erhalten wurden, zeigt, daß nur ein geringer Teil der durch die Einzelfall-Erfassungen bekannten Sterbefälle in die Bundesstatistik eingeht (Abb. 6). Das sind in den neuen Bundesländern und Berlin beispielsweise drei von 16 Fällen im Jahre 1994 bzw. vier von $20 \mathrm{im}$ Jahre 1996 (Angaben des Statistischen Bundesamtes, Sterbefälle nach Ländern und Altersgruppen).

Hierzu ist anzumerken, daß die Zahlen der Bundesstatistik aus der Auswertung der Totenscheine resultieren. Diese berücksichtigen - wie schon früher beschrieben - nur die Fälle, für die der Gasbrand als Grundleiden deklariert wurde [3]. Da es sich bei einem großen Teil der Sterbefälle um Patienten mit anderen Grundleiden handelt - Durchblutungsstörungen, Diabetes, Appendizitis, Malignome usw. - wird der Arzt nicht notwendigerweise den Gasbrand als Grundleiden angeben. Gasbrandfälle, für die andere Grundleiden angegeben werden, entfallen bei der Auswertung durch die Statistischen Landesämter.

Sterbefälle an Gasbrand stehen in der Regel in engem zeitlichen Zusammenhang mit der Diagnostik der Erkrankung. Das scheint auch ein Grund dafür zu sein, daß Gasbrandsterbefälle häufig in den Meldungen nach Bundes-Seuchengesetz erfaßt werden.

Für andere Infektionskrankheiten ist nicht selten der umgekehrte Fall anzutreffen, daß nämlich die Todesursa- chenstatistik mehr Sterbefälle aufweist als die Meldung nach Bundes-Seuchengesetz enthält.

Die Letalität des Gasbrandes, die aus den in verschiedener Weise gewonnenen Daten errechnet wird, differiert ganz erheblich. Zieht man die Zahlen des Statistischen Bundesamtes für die Berechnung der Letalität heran, ergeben sich Letalitätsraten, die für den Zeitraum von 1992 bis 1996 in den einzelnen Jahren zwischen $13,4 \%$ und $19,1 \%$ liegen. Diese sind aus den o. a. Gründen als unrealistisch $\mathrm{zu}$ bewerten. Zieht man die Ergebnisse der Sondererhebungen heran, errechnen sich für die einzelnen Jahre Letalitätsraten, die zwischen $34,6 \%$ und $62,1 \%$ liegen.

Deutliche Unterschiede ergeben sich bei der Berechnung der Letalität der Gasbranderkrankungen unter Berücksichtigung der verschiedenen Vorerkrankungen, Operationen bzw. unfallbedingten Verletzungen (Abb. 7). Wurde für Gasbrandfälle, die in Verbindung mit dem

\section{Zusammenfassende Beurteilung}

Obwohl sich die medizinische Betreuung und Versorgung in den vergangenen Jahren ständig verbessert haben, werden auch heute in Deutschland noch in jedem Jahr zwischen 100 und 140 Erkrankungen an Gasbrand gemeldet. Da die Infektion in vielen Fällen nicht beherrscht werden kann, kommt es noch immer bei einem großen Teil der Erkrankungen zu einem tödlichen Verlauf (rund $52 \%$ in den neuen Bundesländern und Berlin).

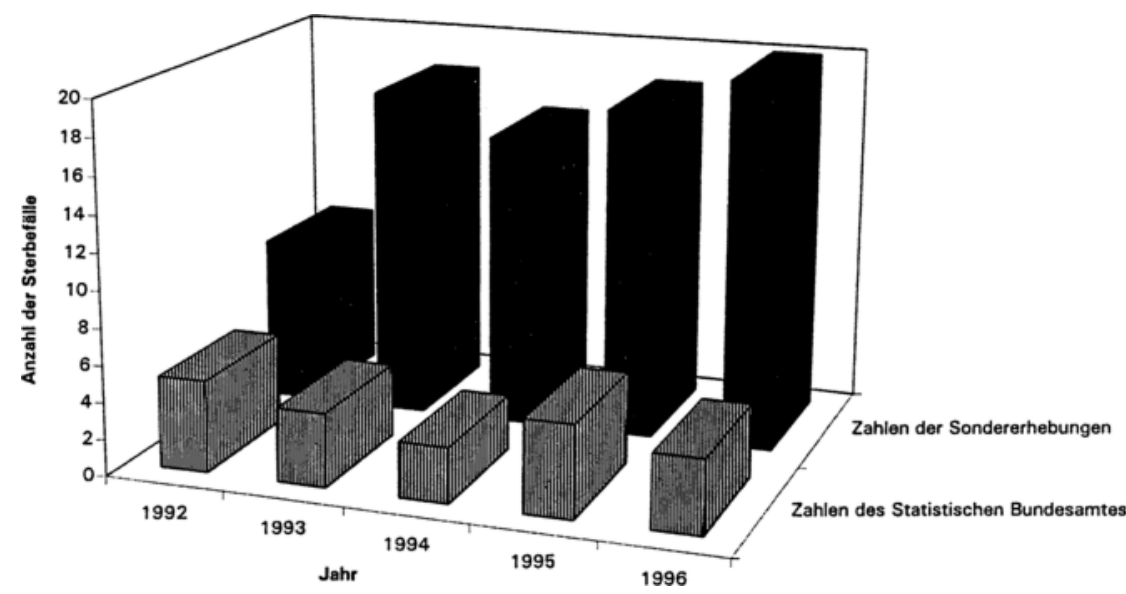

Abbildung 6: Gasbrand in den neuen Bundesländern und Berlin 1992 bis 1996 - Vergleich der Sterbefallzahlen. 


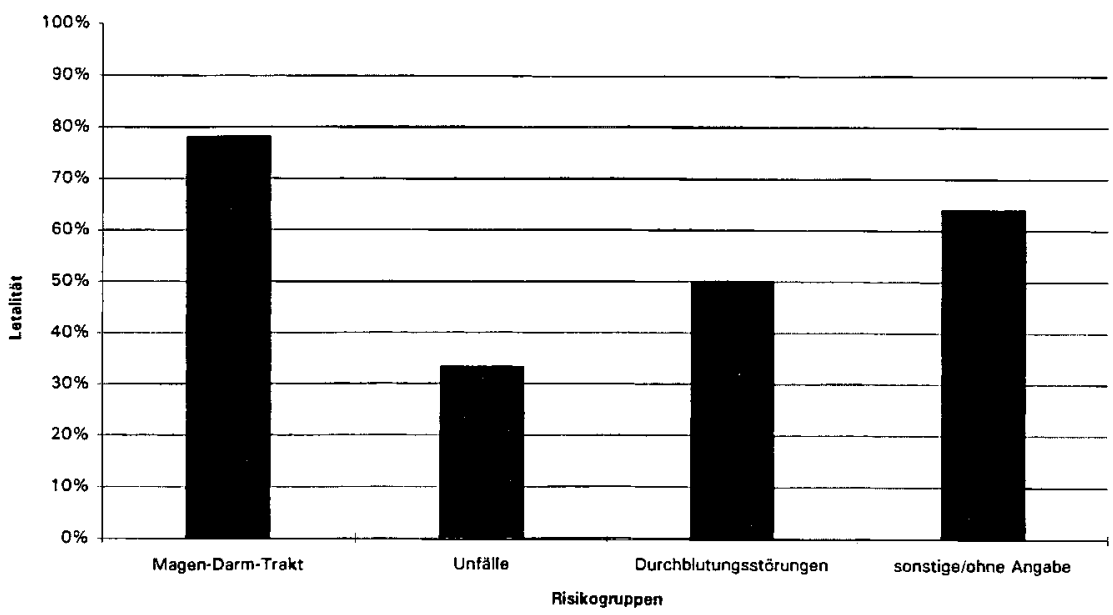

Abbildung 7: Letalität der Gasbranderkrankungen 1992 bis 1997 in den neuen Bundesländern und Berlin.

Die Ergebnisse zeigen, daß Personen nahezu jeder Altersgruppe von Gasbrand betroffen sein können. Bei einem großen Teil der Erkrankten handelt es sich jedoch um ältere Personen, deren Vorerkrankungen besondere Risikofaktoren für das Entstehen einer Gasbranderkrankung darstellen. Betroffen sind aber auch jüngere Personen, die infolge erlittener schwerer Verletzungen (z. B. offene Frakturen) besonders gefährdet sind, an Gasbrand zu erkranken. Bei ihnen ist die Letalität jedoch deutlich niedriger.

gen sind nur wenig Angaben verfügbar. So werden im Rahmen der Meldungen nach Bundes-Seuchengesetz lediglich die Zahlen der Erkrankten, aufgeschlüsselt auf die einzelnen Bundesländer, erfaßt sowie - wie schon erwähnt - in der Todesursachenstatistik - die Anzahl der Sterbefälle. Aus diesem Grunde erschien eine Auswertung der detaillierten Angaben, die aus den neuen Bundesländern und Berlin übermittelt wurden, besonders interessant, zumal für dieses Gebiet schon langjährige Daten vorliegen (seit 1979).

Hinsichtlich der in Deutschland insgesamt auftretenden Gasbranderkrankun-

Auch wenn es bei den Hauptrisikogruppen im Laufe der letzten Jahre zu einer
Verschiebung in der Rangfolge gekommen ist - nunmehr stehen Gasbrandfälle im Zusammenhang mit Durchblutungsstörungen an vorderster Stelle - so blieben doch im Laufe der Jahre die schon seit Beginn der detaillierten Auswertungen ermittelten hauptsächlichen Ursachen für Gasbrand-Erkrankungen insgesamt unverändert.

Dies trug auch zu der Entscheidung bei, $\mathrm{da}$ - unter Berücksichtigung einer notwendig werdenden Beschränkung auf die gegenwärtig wichtigsten Erkrankungsgruppen - zukünftig Einzelfallerfassungen von Gasbrandfällen am RKI in dieser Form nicht weitergeführt werden

Literatur:

[1] Pöhn, H. P., und Rasch, G.: Statistik meldepflichtiger übertragbarer Krankheiten. BGASchriften 5/93. München: MMV Medizin Verlag 1994.

[2] Statistisches Bundesamt: Gesundheitswesen Fachserie 12, Reihe 4: Todesursachen (Wiesbaden 1992-1996).

[3] Zastrow, K.-D., Schöneberg, I., und Rasch, G.: Gasbrand aus der Sicht des Krankenhaushygienikers. Bundesgesundhbl. 39, 1 (1996) 5-8.

[4] Statistisches Bundesamt: Gesundheitswesen, Fachserie 12, Reihe 2: Meldepflichtige Krankheiten (Wiesbaden 1992-1996).
Dr. Gernot Rasch, Dr. Irene Schöneberg, Dr. Lothar Apitzsch, Fachgebiet Meldepflichrige Krankheiten, Robert Koch-Institut, Berlin 